\title{
Environmental TEM Studies of CoMn nanoalloys as Model Fischer-Tropsch Catalysts
}

\author{
Cecile S. Bonifacio ${ }^{1}$, Nikos Liakakos ${ }^{2}$, Miquel Salmeron ${ }^{2}$, and Judith C. Yang ${ }^{1}$ \\ 1. Department of Chemical and Petroleum Engineering, University of Pittsburgh, Pittsburgh, PA \\ 2. Materials Science Division, Lawrence Berkeley National Laboratory, Berkeley, CA
}

Fischer-Tropsch(FT) reaction involves the hydrogenation of carbon monoxide to hydrocarbons typically by group VIII metals [1]. Cobalt is one of the first metals used as FT catalyst and is typically mixed with $\mathrm{Mn}$ to promote carbon monoxide conversion to more valuable higher $\left(\mathrm{C}_{5+}\right)$ hydrocarbons and olefins. The properties of metal catalysts can be controlled by fine-tuning chemical composition, surface oxidation, structure and dimension[2,3]. However, the performance of FT catalysts is strongly affected by oxidizing and reducing pre-treatments[1]. In this case, well-defined size and compositions of CoMn nanoalloys were synthesized and studied in oxidizing and reducing conditions as necessary for its viability as FT catalyst. Here in situ TEM was performed to determine the elemental distributions and morphological changes in the CoMn nanoalloys upon exposure to oxidizing and reducing environments to elucidate the observed nanoalloys' catalytic properties.

Environmental transmission electron microscope (ETEM) experiments of 1 cycle of oxidation and reduction at 0.1 mTorr in $\mathrm{O}_{2}$ and $\mathrm{H}_{2}$ gas at $300^{\circ} \mathrm{C}$ of the CoMn nanoalloys were performed. These nanoalloys (diameter of 3-6 nm) were dispersed in hexane and drop-casted on SiN grids for the in situ studies. A Hitachi H-9500 $\mathrm{LaB}_{6}$ ETEM microscope operated at $200 \mathrm{KV}$ was used for simultaneous acquisitions of videos and high resolution TEM (HRTEM) images. The elemental distributions of Co and $\mathrm{Mn}$ within the particle were obtained through the analysis of the HRTEM image's fast Fourier transform (FFT) by application of annular mask on a given lattice spot on the FFT [4].

Figure 1a shows a HRTEM image of a single NP at room temperature (RT) with the Co and Mn lattice spots separately masked in b. After masking the specific spots and inverting the FFT, the elemental distribution of $\mathrm{Co}$ and $\mathrm{Mn}$ were obtained and superimposed on initial HRTEM image (Figure 1c). Formation of $\mathrm{Co}$ and $\mathrm{Mn}$ oxides were detected after the oxidation cycle (Figure 2a). During oxidation, $\mathrm{MnO}$ is readily formed and appeared to cover the $\mathrm{CoO}$ (light blue areas in Figure 2a). Such results can be attributed to the faster oxidation rates for Mn versus Co resulting to $\mathrm{Mn}^{3+}$ doping of $\mathrm{CoO}$ [5]. Further reduction resulted to Co being reduced to its metallic phase and $\mathrm{Mn}$ remained as oxide. During the reduction cycle, both Co and Mn were equally distributed within the NP (elemental map in Figure 2b).

Additional ETEM experiments with longer exposure times of the reaction cycles are underway with more acquired HRTEM images during the reaction cycles for a quantitative analysis of the nanoalloy's reconstruction. Furthermore, correlation of the nanoalloy's reconstruction from the ETEM studies to ambient pressure X-ray photoelectron spectroscopy (AP-XPS) study of similar experimental conditions along with the catalyst activity experiments will be provided to obtain the optimum reaction conditions of these CoMn nanoalloys as model FT catalysts [6].

References:

[1] A.Y. Khodakov et al, Chemical Reviews 107 (2007), p. 1692

[2] R. Tiruvalam et al, Faraday Discussions 152 (2011), p. 63 
[3] S. Mandal, KM Krishnan, Journal of Materials Chemistry 17 (2007), p. 372

[4] C. Holse et al, The Journal of Physical Chemistry C 119 (2015) p. 2804

[5] M.E. El-Dahshan, Transactions of the Japan Institute of Metals 23 (1982), p. 177

[6] CB and JY acknowledge financial support by DOE Basic Energy Sciences (DE FG02-03ER15476).

NL and MS were supported by the Director, Office of Science, Office of Basic Energy Sciences, Chemical Sciences, Geosciences, and Biosciences Division, under the Department of Energy Contract No. DE-AC02-05CH11231.

(a)

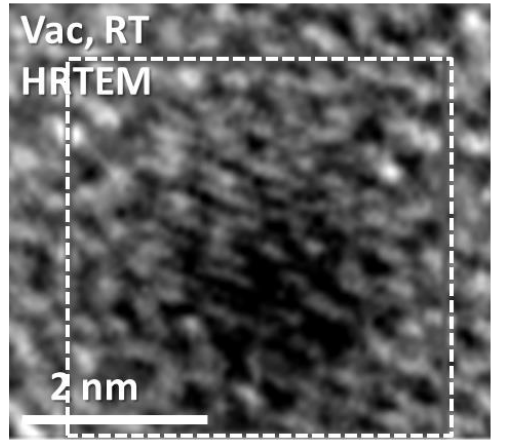

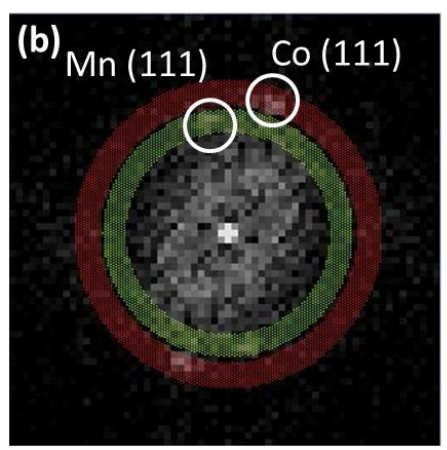

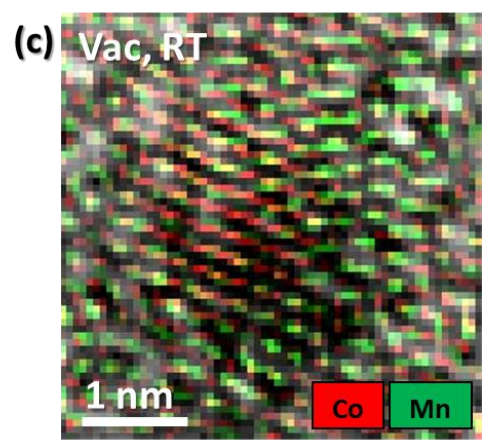

Figure 1. (a) HRTEM image of a single CoMn nanoalloy at room temperature before gas exposure. (b) shows the identified $\mathrm{Co}(111)$ and $\mathrm{Mn}(111)$ spots with applied annular masks on the FFT acquired from the selected area in a. (c) is image (a) with the colored areas of Co (red) and Mn (green) superimposed.

(a)
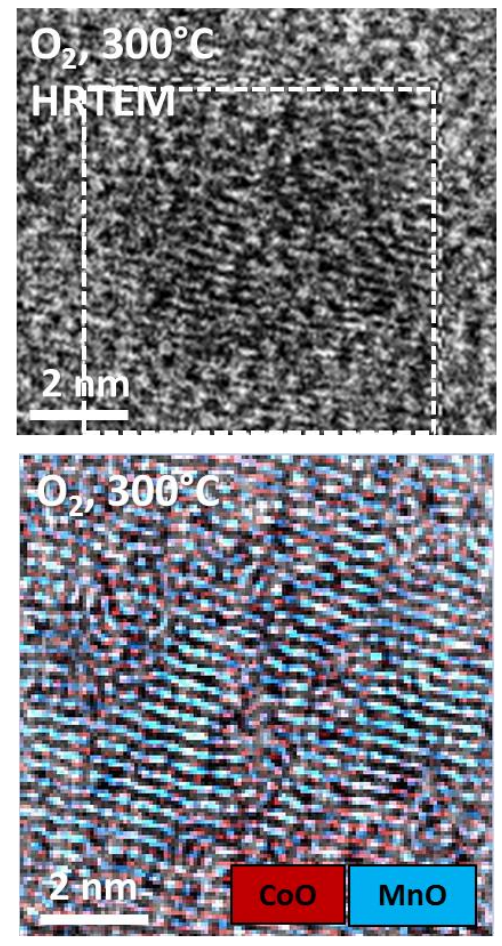

(b)
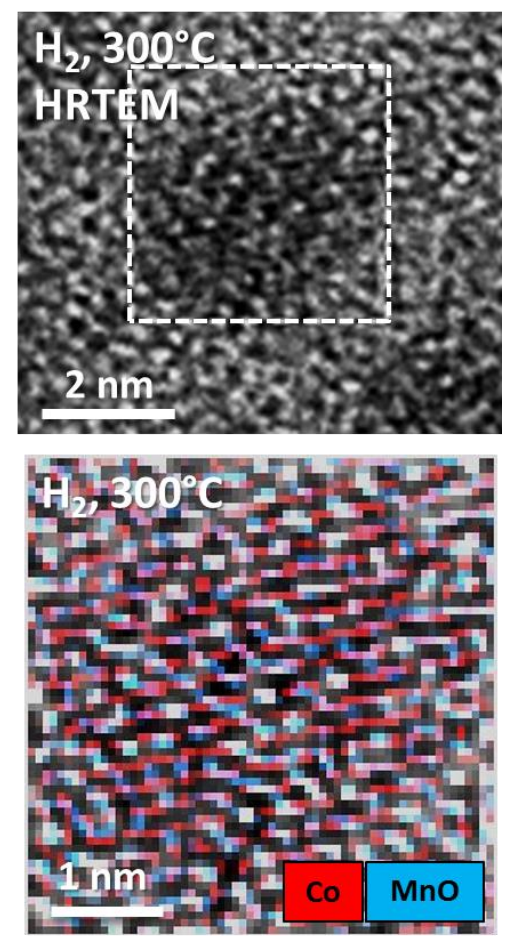

Figure 2. (a) HRTEM image of a single CoMn nanoalloy under $\mathrm{O}_{2}$ exposure at $300^{\circ} \mathrm{C}$ identified with areas of $\mathrm{CoO}$ (maroon) and $\mathrm{MnO}$ (blue). Exposure to $\mathrm{H}_{2}$ at $300^{\circ} \mathrm{C}(\mathbf{b})$ resulted to reduction of $\mathrm{CoO}$ to $\mathrm{Co}$ (red) and intermixing with $\mathrm{MnO}($ blue). 